\title{
Blocker size via matching minors
}

\author{
Nikola Yolov
}

August 9, 2018

\begin{abstract}
Finding the maximum number of maximal independent sets in an $n$ vertex graph $G, i(G)$, from a restricted class is an extensively studied problem. Let $k K_{2}$ denote the matching of size $k$, that is a graph with $2 k$ vertices and $k$ disjoint edges. A graph with an induced copy of $k K_{2}$ contains at least $2^{k}$ maximal independent sets. The other direction was established in a series of papers [Far89, BY89, FHT93 finally yielding $i(G) \leq(n / k)^{2 k}$ for a graph $G$ without an induced $(k+1) K_{2}$. Alekseev proved in Ale07 that $i(G)$ is at most the number of induced matchings of $G$.

This work generalises the aforementioned results to clutters. The right substructures in this setting are minors rather than induced subgraphs. Maximal independent sets of a clutter $\mathcal{H}$ are in one-to-one correspondence to the sets of its blocker, $b(\mathcal{H})$, hence $i(\mathcal{H})=|b(\mathcal{H})|$. We show that

$$
|b(\mathcal{H})| \leq \sum_{m=0}^{k \cdot f(r)}\left(\begin{array}{c}
|\mathcal{H}| \\
m
\end{array}\right)\left(\begin{array}{l}
r \\
2
\end{array}\right)^{m}
$$

for a $(k+1) K_{2}$-minor-free clutter $\mathcal{H}$ where $f(r)=(2 r-3) 2^{r-2}$ and $r$ is the maximum size of a set in $\mathcal{H}$. A key step in the proofs is, similarly to Alekseev's result, showing that $i(\mathcal{H})$ is bounded by the number of a substructure called semi-matching, and then proving a dependence between the number of semi-matchings and the number of minor matchings. Note that similarly to graphs, a clutter containing a $k K_{2}$ minor has at least $2^{k}$ maximal independent sets.

From a computational perspective, a polynomial number of independent sets is particularly interesting. Our results lead to polynomial algorithms for restricted instances of many problems including Set Cover and k-SAT.

Keywords. Transversals, Maximal Independent set, Clutter, Clutter Minor, Induced Matching, Blocker, k-SAT, Set Cover
\end{abstract}

\section{Introduction}

\section{$1.1 \quad$ Previous work}

An independent (or stable) set of a graph $G$ is a subset of its vertices not containing an edge. Let $i(G)$ be the number of inclusion maximal independent 
sets of $G$. The first result on $i(G)$ is by Moon and Moser [MM65 showing that $i(G) \leq 3^{n / 3}$ for an $n$-vertex graph $G$. The exact number has been found for general graphs, connected graphs, trees, forests, triangle-free graphs and other classes. This research sparked the interested of finding a family of classes controlled by a parameter where the maximum number of maximal independent sets can be bounded in terms of the parameter. It is easy to see that a graph $G$ with an induced matching of size $k$ contains at least $2^{k}$ maximal independent sets. Farber proved in Far89 that an $n$-vertex graph $G$ without an induced $2 K_{2}$ has $O\left(n^{2}\right)$ maximal independent sets. Let $\rho=\rho(G)=\mid\{u v \in E(G)$ : $N(u) \cup N(v) \neq V(G)\} \mid$, and let $\nu=\nu(G)$ be the maximal $k$ such that $G$ contains $k K_{2}$ as an induced graph. Balas and Yu generalised the result of Farber in BY89] showing that $i(G) \leq \rho^{\nu}+1$. Farber, Hujter and Tuza proved in FHT93 that $i(G)<\sqrt[3]{\nu} e^{1-\nu}\left(\begin{array}{c}n \\ 2 \nu\end{array}\right)$ for a graph on $n \geq 4 \nu$ vertices. All these results are generalised by an elegant theorem by Alekseev [Ale07] stating that $i(G)$ is at most the number of induced matchings of $G$.

A number of otherwise NP-complete problems can be solved in polynomial time if the list of all maximal stable sets of the underlying graph is given as input, as a trivial example consider the maximum independent set problem. Other problems have their complexity reduced. For instance, given this input, the chromatic number problem can be reduced to the set cover problem, which is $\log n$-approximable, while the chromatic number is not $n^{1-\epsilon}$ approximable for any $\epsilon>0$, assuming NP $\nsubseteq \mathrm{ZPP}$ FK98. The set of maximal independent sets of a graph can be computed in time polynomial with respect to their number JJYP8, and hence graphs with polynomial-sized $i(G)$ are of a particular interest for computer science. A class of graphs $\mathcal{G}$ has polynomially bounded $i(G)$ if there is a polynomial $p(x)$ such that $i(G) \leq p(v(G))$ for every $G \in \mathcal{G}$. The results above imply that a hereditary class of graphs $\mathcal{G}$, that is a class closed under taking induced subgraphs, has polynomially bounded $i(G)$ if and only if $k K_{2} \notin \mathcal{G}$ for some $k$.

\subsection{New results}

The aim of this paper is to generalise these results to hypergraphs. An independent set of a hypergraph $\mathcal{H}$ is a subset of $V(\mathcal{H})$ containing no $S \in \mathcal{H}$. Note that if a hypergraph $H$ contains two hyperedges $g$ and $h$, such that $g \subset h$, in which case we say that $h$ is subsumed by $g$, the set of maximal independent sets of $H$ does not change if $h$ is removed. Therefore subsumed edges play no role here, and hence we lose nothing by assuming there are no such edges. This leads us to the first definition.

Definition 1.1 (Clutter). A set system is a finite set of finite subsets of the natural numbers $\mathbb{N}$. A set system is called clutter, Sperner family or antichain if its sets are incomparable. Every set system $\mathcal{H}$ we associate with a vertex set $V(\mathcal{H}):=\bigcup_{S \in \mathcal{H}} S$ and $\operatorname{rank} r k(\mathcal{H}):=\max _{S \in \mathcal{H}}|S|$. Given a set system $\mathcal{H}$ we define $\operatorname{cl}(\mathcal{H})$ to be the clutter obtained from $\mathcal{H}$ by removing all non-minimal (subsumed) edges. 
The edge clutter of a graph $G$ is the clutter composed of the edges of $G$.

In the context of clutters, the complements of independent sets, called transversals, are more natural to work with.

Definition 1.2 (Blocker). A transversal of a clutter $\mathcal{H}$ is a set intersecting every $S \in \mathcal{H}$. A minimal transversal is a transversal not containing another transversal as a subset. The blocker of $\mathcal{H}$, denoted $b(\mathcal{H})$, is the clutter consisting of all minimal transversals of $\mathcal{H}$.

One reason why transversals are preferred is because $b(b(\mathcal{H}))=\mathcal{H}$ for every clutter $\mathcal{H}$, and this property is not shared with independent sets. For example, take $C_{6}=\{12,23,34,45,56,61\}$ and note that indep $\left(C_{6}\right)=\{14,25,36,135,246\}$, indep $\left(\operatorname{indep}\left(C_{6}\right)\right)=\{123,234,345,456,561,612\} \neq C_{6}$.

Definition 1.3 (Deletion and Contraction). Suppose $\mathcal{H}$ is a clutter and $v \in \mathbb{N}$. Define

$$
\begin{aligned}
& \mathcal{H} \backslash v:=\{S \in \mathcal{H}: v \notin S\} \text { to be } \mathcal{H} \text { with } v \text { deleted, and } \\
& \mathcal{H} / v:=\operatorname{cl}(\{S-v: S \in \mathcal{H}\}) \text { to be } \mathcal{H} \text { with } v \text { contracted. }
\end{aligned}
$$

Definition 1.4 (Minor). We say that $\mathcal{F}$ is a minor of $\mathcal{H}$ if $\mathcal{F}$ can be obtained from $\mathcal{H}$ through a series of deletions and contractions. We write $\mathcal{F} \subseteq m$ H to denote that $\mathcal{F}$ is isomorphic to a minor of $\mathcal{H}$ and say that $\mathcal{H}$ is $\mathcal{F}$-minor-free if $\mathcal{F} \nsubseteq_{m} \mathcal{H}$.

Note that $\mathcal{H} \backslash v=\operatorname{cl}(\mathcal{H} \backslash v)$, and hence all minors of a clutter are clutters.

We are in position to state our main theorem.

Theorem 1.5. Suppose $\mathcal{H}$ is a $(k+1) K_{2}$-minor-free rank $r$ clutter. Then

$$
|b(\mathcal{H})| \leq \sum_{m=0}^{k \cdot(2 r-3) 2^{r-2}}\left(\begin{array}{c}
|\mathcal{H}| \\
m
\end{array}\right)\left(\begin{array}{l}
r \\
2
\end{array}\right)^{m} .
$$

The theorem holds with equality for $\mathcal{H} \cong k K_{2}$. Similarly to the case of graphs, we see that a minor-closed class of clutters has polynomially bounded $|b(\mathcal{H})|$ if and only if it does not contain $k K_{2}$ for some $k$.

\section{Notation and preliminaries}

Denote the set $\{1, \ldots n\}$ by $[n]$. It is often convenient to write $S \cup x$ and $S-x$ instead of $S \cup\{x\}$ and $S \backslash\{x\}$.

We quickly revise some well-known properties of clutters and blockers used throughout the proofs.

Definition 2.1 (Join and Meet). Suppose $\mathcal{H}$ and $\mathcal{F}$ are clutters. We define

$\mathcal{H} \vee \mathcal{F}:=\operatorname{cl}(\mathcal{H} \cup \mathcal{F})$ to be the join of $\mathcal{H}$ and $\mathcal{F}$, and

$\mathcal{H} \wedge \mathcal{F}:=\operatorname{cl}(\{S \cup T: S \in \mathcal{H}, T \in \mathcal{F}\})$ to be the meet of $\mathcal{H}$ and $\mathcal{F}$. 
Lemma 2.2 (Algebraic properties). Let $\mathcal{H}, \mathcal{G}$ and $\mathcal{F}$ be clutters and let $v$ and $u$ be distinct elements of $\mathbb{N}$. The operations defined above have the following properties:

1. deletion and contraction commute, that is $\mathcal{H} \backslash v \backslash u=\mathcal{H} \backslash u \backslash v, \mathcal{H} \backslash v / u=$ $\mathcal{H} / u \backslash v$ and $\mathcal{H} / v / u=\mathcal{H} / u / v$;

2. if we denote the set of all clutters by $C L(\mathbb{N})$, the clutters $\emptyset$ and $\{\emptyset\}$ by $\widehat{0}$ and $\hat{1}$ respectively, then $(C L(\mathbb{N}), \vee, \wedge, \widehat{0}, \hat{1})$ forms a bounded distributive lattice, that is

$$
\begin{gathered}
\text { Commutative laws } \\
\mathcal{F} \vee \mathcal{G}=\mathcal{G} \vee \mathcal{F} \\
\mathcal{F} \wedge \mathcal{G}=\mathcal{G} \wedge \mathcal{F} \\
\\
\text { Identity laws } \\
\mathcal{F} \vee \widehat{0}=\mathcal{F} \\
\mathcal{F} \wedge \widehat{1}=\mathcal{F}
\end{gathered}
$$

$$
\begin{gathered}
\text { Associative laws } \\
\mathcal{F} \vee(\mathcal{G} \vee \mathcal{H})=(\mathcal{F} \vee \mathcal{G}) \vee \mathcal{H} \\
\mathcal{F} \wedge(\mathcal{G} \wedge \mathcal{H})=(\mathcal{F} \wedge \mathcal{G}) \wedge \mathcal{H} \\
\text { Distributive laws } \\
\mathcal{F} \wedge(\mathcal{G} \vee \mathcal{H})=(\mathcal{F} \wedge \mathcal{G}) \vee(\mathcal{F} \wedge \mathcal{H}) \\
\mathcal{F} \vee(\mathcal{G} \wedge \mathcal{H})=(\mathcal{F} \vee \mathcal{G}) \wedge(\mathcal{F} \vee \mathcal{H})
\end{gathered}
$$

Absorption laws
$\mathcal{F} \vee(\mathcal{F} \wedge \mathcal{G})=\mathcal{F}$
$\mathcal{F} \wedge(\mathcal{F} \vee \mathcal{G})=\mathcal{F}$

The lattice has the additional properties that $\mathcal{F} \vee \mathcal{H}=\widehat{1}$ iff $\mathcal{F}=\widehat{1}$ or $\mathcal{H}=\widehat{1}, \mathcal{F} \wedge \mathcal{H}=\widehat{0}$ iff $\mathcal{F}=\widehat{0}$ or $\mathcal{H}=\widehat{0}$, and $b(\widehat{0})=\widehat{1} ;$

3. the map $\mathcal{H} \mapsto b(\mathcal{H})$ is a duality operation: $b(b(\mathcal{H}))=\mathcal{H}$;

4. deletion is the dual operation of contraction and vice versa: $b(\mathcal{H} \backslash v)=$ $b(\mathcal{H}) / v$ and $b(\mathcal{H} / v)=b(\mathcal{H}) \backslash v ;$

5. join is the dual operation of meet and vice versa: $b(\mathcal{H} \vee \mathcal{F})=b(\mathcal{H}) \wedge b(\mathcal{F})$ and $b(\mathcal{H} \wedge \mathcal{F})=b(\mathcal{H}) \vee b(\mathcal{F})$;

6. the property of being a minor is preserved by duality: $\mathcal{F} \subseteq \mathcal{M} \mathcal{H}$ if and only if $b(\mathcal{F}) \subseteq \mathcal{M} b(\mathcal{H})$;

7. minors commute with join and meet:

$$
(\mathcal{H} \vee \mathcal{F}) \backslash v=\mathcal{H} \backslash v \vee \mathcal{F} \backslash v \quad \text { and } \quad(\mathcal{H} \vee \mathcal{F}) / v=\mathcal{H} / v \vee \mathcal{F} / v
$$

Given a $k$-set $S$, the notation $\mathcal{H} \backslash S$ stands for $\mathcal{H} \backslash s_{1} \ldots \backslash s_{k}$, where $\left\{s_{1}, \ldots s_{k}\right\}$ is an arbitrary ordering of $S$. The choice of the ordering does not matter by Lemma 2.2 (1). We define $\mathcal{H} / S$ to be $\mathcal{H} / s_{1} \ldots / s_{k}$ likewise. Finally, given disjoint sets $S$ and $T$ we define $\mathcal{H}[S ; T]$ to be $\mathcal{H} \backslash S / T$. This operation has the nice property that $\mathcal{H}\left[S_{1} \cup S_{2} ; T_{1} \cup T_{2}\right]=\mathcal{H}\left[S_{1} ; T_{1}\right]\left[S_{2} ; T_{2}\right]$ for any four sets $S_{1}$, $S_{2}, T_{1}$ and $T_{2}$ such that $\left(S_{1} \cup S_{2}\right) \cap\left(T_{1} \cup T_{2}\right)=\emptyset$, which follows from Lemma 2.2 (1). We stress the trivial fact that $\mathcal{F} \subseteq m \mathcal{H}$ iff $\mathcal{F} \cong \mathcal{H}[S ; T]$ for some $S$ and $T$. 


\section{Outline of the results}

Definition 3.1 (Semi-matching and expanded minor matching). Consider a set $\mathcal{S}$ of the form $\left\{\left(L_{i}, S_{i}\right)\right\}_{i=1}^{k}$, a clutter $\mathcal{H}$ and the following conditions:

1. $\left|L_{i}\right|=2, L_{i} \subseteq S_{i}$ and $S_{i} \in \mathcal{H}$ for each $1 \leq i \leq k$,

2. $\left\{L_{i}\right\}_{i=1}^{k}$ are pairwise disjoint,

3a. $L_{i} \nsubseteq \subseteq S_{j}$ for $i \neq j$,

3b. $L_{i} \cap S_{j}=\emptyset$ for $i \neq j$,

4. all sets $S \in \mathcal{H}$ contained in $\bigcup_{i} S_{i}$ contain as a subset at least one set $L_{i}$.

We call $\mathcal{S}$ a semi-matching if it satisfies properties 1, 2, 3a and 4; and an expanded minor matching if it additionally satisfies $3 b$.

Semi-matchings and expanded minor matchings coincide with induced matchings in edge clutters.

The following theorem is a generalisation of Alekseev's theorem from Ale07. to clutters.

Theorem 3.2 (Decomposition theorem). For every clutter $\mathcal{H},|b(\mathcal{H})|$ is at most the number of semi-matchings of $\mathcal{H}$.

Note the lack of restrictions on the rank of $\mathcal{H}$.

The following lemma establishes that extended minor matchings and minor matchings are essentially equivalent.

Lemma 3.3. If $\mathcal{S}=\left\{\left(L_{i}, S_{i}\right)\right\}_{i=1}^{k}$ is an expanded minor matching of a clutter $\mathcal{H}$, then $\left\{L_{i}\right\}_{i=1}^{k} \cong k K_{2}$ is a minor of $\mathcal{H}$. Conversely, if $\left\{L_{i}\right\}_{i=1}^{k}$ is a $k K_{2}$ minor of $H$, we can find sets $\left\{S_{i}\right\}_{i=1}^{k}$ such that $\left\{\left(L_{i}, S_{i}\right)\right\}_{i=1}^{k}$ is an extended minor matching of $\mathcal{H}$.

Proof. To prove the first part, contract $\left(\bigcup_{i=1}^{k} S_{i}\right) \backslash\left(\bigcup_{i=1}^{k} L_{i}\right)$ and delete $V(\mathcal{H}) \backslash$ $\left(\bigcup_{i=1}^{k} S_{i}\right)$. Any non-deleted edge is subsumed by some $L_{i}$.

For the second part, each $L_{i}$ must be a contraction of a set $S_{i} \in \mathcal{H}$. It is a routine to verify that $\left\{\left(L_{i}, S_{i}\right)\right\}_{i=1}^{k}$ is an extended minor matching of $\mathcal{H}$.

Clearly every extended minor matching is a semi-matching. In the opposite direction, we see that, in clutters of bounded rank, semi-matchings contain expanded minor matchings as subsets of linear size.

Theorem 3.4 (Matching theorem). Suppose $\mathcal{H}$ is a clutter of rank at most $r$ and $\mathcal{S}$ is a semi-matching of $\mathcal{H}$. There is an expanded minor matching $\mathcal{S}^{\prime} \subseteq \mathcal{S}$ of size at least $|\mathcal{S}| 2^{-(r-2)} /(2 r-3)$. 
Theorem 1.5 follows directly from the matching and decomposition theorems and Lemma 3.3. Theorem 3.4 is sharp in the sense that if we put $r=2$, we see that $\mathcal{S} \equiv \mathcal{S}^{\prime}$. In this case the entire $\mathcal{S}$ corresponds to an induced matching (in graph theoretical sense).

The matching theorem does not hold for clutters of unbounded rank. Consider $\mathcal{H}$ over the vertex set $\left\{a_{1}, \ldots, a_{n}\right\} \cup\left\{b_{1}, \ldots, b_{n}\right\}$ defined by $\mathcal{H}=\left\{S_{i}\right\}_{i=1}^{n}$, where $S_{i}=\left\{a_{i}\right\} \cup\left\{b_{j}: 1 \leq j \leq i\right\}$. We see that $\mathcal{H}$ contains a semi-matching $\mathcal{S}=\left\{\left(a_{i} b_{i}, S_{i}\right)\right\}_{i=1}^{n}$ of size $n$, but $\mathcal{H}$ is $2 K_{2}$-minor-free.

\section{Decomposition and Matching theorems}

We now present the main technical tool to handle semi-matchings.

Definition 4.1 (Expansion). Suppose $R_{1}, \ldots, R_{k}, C$ are subsets of $\mathbb{N}$ such that $\left\{R_{i}\right\}$ are pairwise disjoint and $\bigcup_{i} R_{i} \subseteq C$. We denote the set of functions $f:\left\{R_{i}\right\}_{i=1}^{k} \rightarrow \bigcup_{i=1}^{k} R_{i}$ such that $f\left(R_{i}\right) \in R_{i}$ for each $i$ by $\mathscr{F}\left(\left\{R_{i}\right\}_{i=1}^{k}\right)$. For each such function $f \in \mathscr{F}\left(\left\{R_{i}\right\}_{i=1}^{k}\right)$ define $i_{f}:=\left\{f\left(R_{i}\right): 1 \leq i \leq k\right\}$ to be the image of $f$. For every clutter $\mathcal{H}$ we define

$$
\mathcal{H} \circ\left(\left\{R_{i}\right\}_{i=1}^{k}, C\right):=\bigvee_{f \in \mathscr{F}\left(\left\{R_{i}\right\}\right)} \mathcal{H}\left[i m_{f} ; C \backslash i m_{f}\right]
$$

Note that $\mathcal{H} \circ\left(\left\{R_{i}\right\}_{i=1}^{k}, C\right) \neq \widehat{1}$ iff $C \backslash i m_{f}$ is independent (does not contain any $S \in \mathcal{H})$ for each $f \in \mathscr{F}\left(\left\{R_{i}\right\}_{i=1}^{k}\right)$, and hence condition 4 of the definition of semi-matching is equivalent to $\mathcal{H} \circ\left(\left\{L_{i}\right\}_{i=1}^{k}, \bigcup_{i=1}^{k} S_{i}\right) \neq \widehat{1}$. We normally shorten $\mathcal{H} \circ\left(\left\{L_{i}\right\}_{i=1}^{k}, \bigcup_{i=1}^{k} S_{i}\right)$ to $\mathcal{H} \circ \mathcal{S}$.

Lemma 4.2. Suppose $\mathcal{H}$ is a clutter, $T \in b(\mathcal{H})$ and $v \in V(\mathcal{H})$. Either $T-v \in$ $b(\mathcal{H} \backslash v)$ or there is $S \in \mathcal{H}$ and $u \in S$ such that $T \backslash\{v, u\} \in b(\mathcal{H} \circ(\{\{v, u\}\}, S))$ and $v \in S-u$.

Proof. Suppose $T-v \notin b(\mathcal{H} \backslash v)=b(\mathcal{H}) / v$. It follows that $v \notin T$ and that there is $T^{\prime} \in b(\mathcal{H})$ such that $T^{\prime}-v \subsetneq T$. Let $u \in T \backslash T^{\prime}$. The key observation here is that

$$
\text { for any } F \in \mathcal{H} \text { either } F \cap T \neq\{u\} \text { or } v \in F \text {. }
$$

Indeed, if $F \cap(T \cup v)=\{u\}$, then $F \cap T^{\prime}=\emptyset$, a contradiction because $T^{\prime}$ is a transversal. Furthermore, we can find a set $S \in \mathcal{H}$ such that $S \cap T=\{u\}$, because $T$ is minimal, and hence $v \in S$ by (11).

We claim that $T-u=T \backslash\{u, v\} \in b(\mathcal{H} \circ(\{\{v, u\}\}, S))$. Indeed,

$$
\begin{aligned}
b(\mathcal{H} \circ(\{\{v, u\}\}, S)) & =b(\mathcal{H}[u ; S-u] \vee \mathcal{H}[v ; S-v]) \\
& =b(\mathcal{H})[S-u ; u] \wedge b(\mathcal{H}[v ; S-v]) .
\end{aligned}
$$

First note that $T-u \in b(\mathcal{H})[S-u ; u]$, because $T \in b(\mathcal{H})$ and $S \cap T=\{u\}$. From (11) we see that $T-u$ intersects all sets in $\mathcal{H} \backslash v$, hence there is $\widetilde{T} \in b(\mathcal{H} \backslash v)$ 
such that $\widetilde{T} \subseteq T-u$. Furthermore, $\widetilde{T} \cap S-v=\emptyset$, so $\widetilde{T} \in b(\mathcal{H} \backslash v) \backslash(S-v)$ $=b(\mathcal{H}[v ; S-v])$. We deduce that $T-u \in b(\mathcal{H})[S-u ; u]$ and $\widetilde{T} \in b(\mathcal{H}[v ; S-v])$, so by the definition of meet $(\wedge)$ there is a set $R \subseteq(T-u) \cup \widetilde{T}=T-u$ such that $R \in b(\mathcal{H})[S-u ; u] \wedge b(\mathcal{H}[v ; S-v])$. However, if $R \neq T-u$, then $R$ is not a transversal of $b(\mathcal{H})[S-u ; u]$, because $T-u$ is a minimal such transversal, in contradiction with the definition of meet.

Lemma 4.3. Suppose $\mathcal{H}$ is a clutter, $C \in \mathcal{H}, R \subseteq C,|R|=2$ and $\mathcal{S}^{\prime}=$ $\left\{\left(L_{i}, S_{i}^{\prime}\right)\right\}_{i=1}^{k}$ is a semi-matching in $\mathcal{H}^{\prime}=\mathcal{H} \circ(\{R\}, C)$. Then there is a semimatching $\mathcal{S}=\left\{\left(L_{i}, S_{i}\right)\right\}_{i=1}^{k} \cup(\{R\}, C)$ in $\mathcal{H}$, such that $\mathcal{H} \circ \mathcal{S}=\mathcal{H}^{\prime} \circ \mathcal{S}^{\prime}$ and $S_{i}^{\prime} \subseteq S_{i} \subseteq S_{i}^{\prime} \cup C$ for each $i$. Pick one such semi-matching, say the lex-first one, and call it $\operatorname{ext}\left(\mathcal{S}^{\prime}, \mathcal{H}, R, C\right)$.

Proof. It follows from the definition of $\mathcal{H} \circ(\{R\}, C)$ that since $S_{i}^{\prime} \in \mathcal{H}^{\prime}$ there is at least one set $S_{i} \in \mathcal{H}$ such that $S_{i}^{\prime} \subseteq S_{i} \subseteq S_{i}^{\prime} \cup C$ and $R \nsubseteq S_{i}$ for each $i \in[k]$. Fix an arbitrary such $S_{i}$ for each $i$ and consider $\mathcal{S}=\left\{\left(L_{i}, S_{i}\right)\right\}_{i=1}^{k} \cup\{(\{R\}, C)\}$. From the definitions it immediately follows that $\mathcal{S}$ satisfies conditions 1, 2 and 3a. To see that $\mathcal{S}$ satisfies 4 expand

$$
\begin{aligned}
\mathcal{H} \circ \mathcal{S} & =\bigvee_{f \in \mathscr{F}\left(\left\{L_{i}\right\} \cup\{R\}\right)} \mathcal{H}\left[i m_{f} ;\left(\cup_{i} S_{i}^{\prime} \cup C\right) \backslash i m_{f}\right] \\
& =\bigvee_{f^{\prime} \in \mathscr{F}\left(\left\{L_{i}\right\}\right)} \bigvee_{r \in R}\left(\mathcal{H}[r ; C-r]\left[i m_{f^{\prime}} ; \cup_{i} S_{i}^{\prime} \backslash i m_{f^{\prime}}\right]\right) \\
& =\bigvee_{f^{\prime} \in \mathscr{F}\left(\left\{L_{i}\right\}\right)}\left(\bigvee_{r \in R} \mathcal{H}[r ; C-r]\right)\left[i m_{f^{\prime}} ; \cup_{i} S_{i}^{\prime} \backslash i m_{f^{\prime}}\right]=\mathcal{H}^{\prime} \circ \mathcal{S}^{\prime} \neq \widehat{1} .
\end{aligned}
$$

Proof of the Decomposition Theorem [3.2. We denote the set of all semi-matchings of a clutter $\mathcal{H}$ by $\operatorname{sm}(\mathcal{H})$. We prove by induction on $|V(\mathcal{H})|$ that $|b(\mathcal{H})| \leq$ $|\operatorname{sm}(\mathcal{H})|$. If $|V(\mathcal{H})|=0$, then $|b(\mathcal{H})| \leq 1$ and $\operatorname{sm}(\mathcal{H})=\{\emptyset\}$. Now suppose $v \in V(\mathcal{H})$. Let $\mathcal{R}$ be the set of pairs $(R, C)$ such that $C \in \mathcal{H}, R \subseteq C,|R|=2$ and $v \in R$. We define $E_{R, C}$ to be the set

$$
E_{R, C}:=\left\{\operatorname{ext}\left(\mathcal{S}^{\prime}, \mathcal{H}, R, C\right): \mathcal{S}^{\prime} \in \operatorname{sm}(\mathcal{H} \circ(\{R\}, C))\right\} .
$$

We see that $E_{R, C} \subseteq \operatorname{sm}(\mathcal{H})$ and $E_{R, C} \cap E_{R^{\prime}, C^{\prime}}=\emptyset$ for $(R, C) \neq\left(R^{\prime}, C^{\prime}\right)$ as they disagree on the pair of sets containing $v$. Furthermore, $\operatorname{sm}(\mathcal{H} \backslash v) \subseteq \operatorname{sm}(\mathcal{H})$ and $\operatorname{sm}(\mathcal{H} \backslash v)$ is disjoint from each $E_{R, C}$ because $v \notin L$ for each $(L, S) \in \mathcal{S} \in$ $\operatorname{sm}(\mathcal{H} \backslash v)$ and every $\mathcal{S} \in E_{R, C}$ contains a pair, $(R, C)$, including $v$. Now we see

$$
\begin{aligned}
|b(\mathcal{H})| & \leq|b(\mathcal{H} \backslash v)|+\sum_{(R, C) \in \mathcal{R}}|b(\mathcal{H} \circ(\{R\}, C))| \\
& \leq|\operatorname{sm}(\mathcal{H} \backslash v)|+\sum_{(R, C) \in \mathcal{R}}|\operatorname{sm}(\mathcal{H} \circ(\{R\}, C))| \\
& =|\operatorname{sm}(\mathcal{H} \backslash v)|+\sum_{(R, C) \in \mathcal{R}}\left|E_{R, C}\right| \leq|\operatorname{sm}(\mathcal{H})| .
\end{aligned}
$$


Proof of the Matching Theorem 3.4. Suppose $\mathcal{S}=\left\{\left(L_{i}, S_{i}\right)\right\}_{i=1}^{\ell}$ is a semi-matching in a rank $r$ clutter $\mathcal{H}$ and let $G$ be a graph over [ $\ell]$ where $i$ is connected to $j$ if $\left|S_{i} \cap L_{j}\right|=1$ or $\left|S_{j} \cap L_{i}\right|=1$. It follows that $G$ contains at most $(r-2) \ell$ edges. A classic result in graph theory states that $\alpha(G) \geq \frac{v(G)^{2}}{2 e(G)+v(G)}$, and hence $G$ contains an independent set $I$ of size at least $\ell /(2 r-3)$.

For $f \in \mathscr{F}\left(\left\{L_{i}\right\}_{i \in[\ell] \backslash I}\right)$ define

$$
I^{\prime}(f):=\left\{i \in I: f\left(L_{j}\right) \notin S_{i} \text { for each } j \in[\ell] \backslash I\right\} .
$$

Sample $g$ uniformly at random from $\mathscr{F}\left(\left\{L_{i}\right\}_{i \in[\ell] \backslash I}\right)$. We have $\mathbb{E}\left|I^{\prime}\right| \geq|I| 2^{-(r-2)} \geq$ $\ell 2^{-(r-2)} /(2 \ell-3)$, so there must be some $J=I^{\prime}(g)$ of at least this size, where $g \in \mathscr{F}\left(\left\{L_{i}\right\}_{i \in[\ell] \backslash I}\right)$.

We claim that $\mathcal{S}_{J}:=\left\{\left(L_{i}, S_{i}\right)\right\}_{i \in J}$ is an extended minor matching. Properties 1 and 2 are inherited from $\mathcal{S}$. Property 3 b holds because $J \subseteq I$ is stable in $G$. Let $h \in \mathscr{F}\left(\left\{L_{i}\right\}_{i \in[\ell] \backslash J}\right)$ be an arbitrary extension of $g$ and, let $C:=\bigcup_{i=1}^{k} S_{i}$ and $C_{J}:=\bigcup_{i \in J} S_{i}$. We see that $C_{J}$ is disjoint from $i m_{h}$. Since every $S \in \mathcal{H}$ with $S \subseteq C$ contains $L_{i}$ as a subset for some $i \in[l]$, it follows that if $S \subseteq C_{J} \subseteq C \backslash i m_{h}$, then $L_{i} \subseteq S$ for some $i \in J$.

\section{Applications}

Often instances $I$ of hard problems correspond to a clutter $\mathscr{H}(I)$, and the solution of $I$ can be read from the blocker of $\mathscr{H}(I)$. Whether or not $b(\mathcal{H})$ can be computed in polynomial time from $\mathcal{H}$ with respect to $|\mathcal{H}|+|b(\mathcal{H})|$ is an open problem, but moreover $|b(\mathscr{H}(I))|$ itself can be exponential. In this paper we gave a sufficient condition for $|b(\mathcal{H})|$ to be polynomial, namely $r k(\mathcal{H}) \leq r$ and $k K_{2} \unlhd_{m} \mathcal{H}$. Furthermore, $b(\mathcal{H})$ can be computed in polynomial time with respect to $|\mathcal{H}|+|b(\mathcal{H})|$ for clutters $\mathcal{H}$ of bounded rank EGM03.

More formally, given a problem $\mathscr{P}$ and a function $\mathscr{H}$ mapping the instances of $\mathscr{P}$ to clutters and computable in polynomial time, we say that the solutions of $(\mathscr{P}, \mathscr{H})$ can be read from the blocker if there is an algorithm that given input $I$ and $b(\mathscr{H}(I))$ determines whether or not $I$ is a "yes" instance in time polynomial with respect to the size of its input.

As a first example, suppose $I$ is an instance of the set cover problem, let $G(I)$ be the hypergraph obtained from $I$ by interchanging the roles of the vertices and the edges, preserving incidence, and let $\mathscr{H}(I):=\operatorname{cl}(G(I))$. We see that the sets of $b(\mathscr{H}(I))$ correspond to minimal covers, and hence a minimum sized cover of $I$ can be easily found from $b(\mathscr{H}(I))$. A minimum weighted cover can be found the same way, and more generally, a minimum cover for a monotone oracle $Q$, that is $Q(S) \leq Q(T)$ if $S \subseteq T$, can be found in polynomial time from $b(\mathscr{H}(I))$.

Another example is the satisfiability problem (SAT). On instance $I$ with variables $X$ and clauses $C$ create a clutter $\mathscr{H}(I)$ with vertices $v_{i}$ and $\bar{v}_{i}$ for each variable $x_{i} \in X$ and a set $S \in \mathscr{H}(I)$ corresponding to the vertices of $c$ for each 
clause $c \in C$. For a satisfying assignment $\sigma: X \rightarrow\{0,1\}$ consider the set

$$
S_{\sigma}=\left\{v_{i}: \sigma\left(x_{i}\right)=1\right\} \cup\left\{\bar{v}_{i}: \sigma\left(x_{i}\right)=0\right\} .
$$

It is clear that $S_{\sigma}$ is a transversal of $\mathscr{H}(I)$. Moreover, every transversal intersecting each pair $\left\{v_{i}, \bar{v}_{i}\right\}$ at most once can be extended to a transversal of the form $S_{\sigma}$ for a satisfying assignment $\sigma$. It follows that $I$ is satisfiable if and only if $b(\mathscr{H}(I))$ contains a set not containing both $v_{i}$ and $\bar{v}_{i}$ for all $x_{i} \in X$.

The aim is not to give a complete list of applications, as there is an abundance of problems whose solutions can be naturally read from the blocker. For nontrivial applications of transversal enumeration in artificial intelligence, machine learning, data mining, model-based diagnosis see [EG02]. The solution of each of these problems can be read from the blocker.

Finally, let $\mathcal{C}_{r, k}$ be the class of $k K_{2}$-minor-free clutters of rank at most $r$. We conclude that given a problem $(\mathscr{P}, \mathscr{H})$ whose solutions can be read from the blocker and positive integers $r$ and $k$, there is a polynomial-time algorithm to solve $\mathscr{P}$ for instances restricted to $\mathscr{H}^{-1}\left(\mathcal{C}_{r, k}\right)$.

The choice for $\mathscr{H}$ is important, as it affects the size of $\mathscr{H}^{-1}\left(\mathcal{C}_{r, k}\right)$. For instance, in the example with satisfiability, if we had added additional sets $\left\{v_{i}, \bar{v}_{i}\right\}$ for each $x_{i} \in X$, and asked for a transversal of size $|X|$, we would have created a large artificial minor matching in most instances, and hence greatly reduced the size of $\mathscr{H}^{-1}\left(\mathcal{C}_{r, k}\right)$.

It is worth noting that for fixed $r$ and $k$ it is possible to test in polynomial time if $\mathcal{H} \in \mathcal{C}_{r, k}$. Indeed, suppose $\left\{\left(L_{i}, S_{i}\right)\right\}_{i=1}^{k}$ is an extended minor matching in $\mathcal{H}$ and $n=V(\mathcal{H})$. Now let $A=\bigcup_{i=1}^{k} L_{i}, B=\bigcup_{i=1}^{k} S_{i} \backslash A$ and observe that

$$
\mathcal{H}[V(\mathcal{H}) \backslash(A \cup B) ; B] \cong k K_{2} ;
$$

and that $|A|=2 k,|B| \leq(r-2) k$. Therefore, to test if $k K_{2} \subseteq_{m} \mathcal{H}$, it suffices to test for $O\left(n^{r k}\right)$ pairs $(S, T)$ if $\mathcal{H}[S ; T] \cong k K_{2}$. The clutter $\mathcal{H}[S ; T]$ can be computed via a naive algorithm in $O\left(|\mathcal{H}|^{2} n\right)$ time.

\section{References}

[Ale07] Vladimir Evgenevich Alekseev. An upper bound for the number of maximal independent sets in a graph. Discrete Mathematics and Applications dma, 17(4):355-359, 2007.

[BY89] Egon Balas and Chang Sung Yu. On graphs with polynomially solvable maximum-weight clique problem. Networks, 19(2):247-253, 1989.

[EG02] Thomas Eiter and Georg Gottlob. Hypergraph transversal computation and related problems in logic and AI. 2002.

[EGM03] Thomas Eiter, Georg Gottlob, and Kazuhisa Makino. New results on monotone dualization and generating hypergraph transversals. SIAM Journal on Computing, 32(2):514-537, 2003. 
[Far89] Martin Farber. On diameters and radii of bridged graphs. Discrete Mathematics, 73(3):249-260, 1989.

[FHT93] Martin Farber, Mihály Hujter, and Zsolt Tuza. An upper bound on the number of cliques in a graph. Networks, 23(3):207-210, 1993.

[FK98] Uriel Feige and Joe Kilian. Zero knowledge and the chromatic number. Journal of Computer and System Sciences, 57(2):187 - 199, 1998.

[JYP88] David S Johnson, Mihalis Yannakakis, and Christos H Papadimitriou. On generating all maximal independent sets. Information Processing Letters, 27(3):119-123, 1988.

[MM65] John W Moon and Leo Moser. On cliques in graphs. Israel journal of Mathematics, 3(1):23-28, 1965. 